\title{
Exploring the effects of lifestyle on breast cancer risk, age at diagnosis, and survival: the EBBA-Life study
}

\author{
Trygve Lofterød ${ }^{1}(1) \cdot$ Hanne Frydenberg ${ }^{1} \cdot$ Vidar Flote $^{1} \cdot$ Anne Elise Eggen $^{2} \cdot$ Anne McTiernan $^{3} \cdot$ Elin S. Mortensen ${ }^{4}$. \\ Lars A. Akslen ${ }^{5,6}$. Jon B. Reitan ${ }^{1} \cdot$ Tom Wilsgaard $^{2} \cdot$ Inger Thune $^{1,7}$
}

Received: 27 January 2020 / Accepted: 9 May 2020 / Published online: 20 May 2020

(c) The Author(s) 2020

\begin{abstract}
Purpose Whether an unfavorable lifestyle not only affects breast cancer risk, but also influences age at onset of breast cancer and survival, is under debate.

Methods In a population-based cohort, the Energy Balance and Breast Cancer Aspects throughout life (EBBA-Life) study, a total of 17,145 women were included. During follow-up, 574 women developed invasive breast cancer. Breast cancer cases were followed for an additional 9.1 years. Detailed medical records were obtained. Cox's proportional hazard regression models were used to study the association between pre-diagnostic lifestyle factors (weight, physical activity, alcohol use, smoking, and hypertension), breast cancer risk, age at diagnosis, and survival.

Results At study entry, $34.3 \%$ of the participating women were overweight and $30.7 \%$ were physically inactive. Mean age at breast cancer diagnosis was 58.0 years, and $78.9 \%$ of the tumors were estrogen receptor positive. Among menopausal women who did not use hormone therapy and had an unfavorable lifestyle (3-5 unfavorable factors), compared with women who had a favorable lifestyle, we observed a twofold higher risk for postmenopausal breast cancer (hazard ratio [HR] 2.13, 95\% confidence interval [CI] 1.23-3.69), and they were 3.4 years younger at diagnosis (64.8 versus 68.2 years, $P=0.032$ ). Breast cancer patients with an unfavorable lifestyle, compared with patients with a favorable lifestyle, had almost a two times higher overall mortality risk (HR 1.96, 95\% CI 1.01-3.80).
\end{abstract}

Conclusions Our study supports a healthy lifestyle improving breast cancer prevention, postponing onset of disease, and extending life expectancy among breast cancer patients.

Keywords Lifestyle $\cdot$ Breast cancer $\cdot$ Onset $\cdot$ Risk $\cdot$ Survival

Electronic supplementary material The online version of this article (https://doi.org/10.1007/s10549-020-05679-2) contains supplementary material, which is available to authorized users.

Trygve Lofterød

tlofteroed@ hotmail.com

1 Department of Oncology, Oslo University Hospital, Oslo, Norway

2 Faculty of Health Services, Institute of Community Medicine, University of Troms $\emptyset$, Troms $\emptyset$, Norway

3 Public Health Sciences Division, Fred Hutchinson Cancer Research Center, Seattle, USA

\section{Introduction}

A global increase in breast cancer incidence has been observed, with incidence rates being almost fourfold higher in the developed than in the less-developed countries, emphasizing that large differences in lifestyle may have an important role to play [1]. Of note, the rates of estrogen receptor (ER)-positive breast cancer incidence are increasing

4 Department of Pathology, University Hospital of North Norway, Troms $\varnothing$, Norway

5 Department of Clinical Medicine, Centre for Cancer Biomarkers CCBIO, University of Bergen, Bergen, Norway

6 Department of Pathology, Haukeland University Hospital, Bergen, Norway

7 Faculty of Health Services, Institute of Clinical Medicine, University of Troms $\emptyset$, Troms $\varnothing$, Norway 
[2], suggesting that an estrogen-dependent mechanism links unfavorable lifestyle to this most common breast cancer subtype. Furthermore, life expectancy for women who have survived breast cancer has been observed to be shorter compared with women in the general population [3], but whether lifestyle factors play a key role in relation to breast cancer survival remains ambiguous.

The association between body composition, including body weight and breast cancer risk, is well known. High Body Mass Index (BMI) has been positively associated with postmenopausal breast cancer [4,5], but most studies report that excess body weight is inversely related to premenopausal breast cancer risk [6]. Obesity has also been associated with reduced breast cancer survival [7]. A physically active lifestyle compared with a sedentary lifestyle has been observed to reduce both pre- and postmenopausal breast cancer risk overall $[8,9]$. The association between physical activity and breast cancer survival is still being debated [9]. The most consistent dietary risk factor for breast cancer is alcohol $[10,11]$. Alcohol intake has been associated with higher estradiol levels and higher mammographic density [12], and both pre- and postmenopausal breast cancer risk $[13,14]$. Recent meta-analysis observed a positive association between hypertension and breast cancer risk $[15,16]$. Moreover, a new cohort study reported a positive association between hypertension and breast cancer mortality after adjustments [17], supporting hypertension as an independent risk factor linked to breast cancer. Tobacco smoking possesses potential mammary carcinogens [18], and recently tobacco use showed an increased risk for ER-positive breast cancer [19].

Possible biological mechanisms operating for most of these lifestyle factors may be mediated by adipose tissue, with low-grade chronic inflammation creating an environment that promotes breast cancer development and growth [20-22]. Another potential mechanism linking these factors is via increased estrogen levels, a key component in breast cancer development [9]. In addition, both hypertension and breast cancer development may act through activation of the renin-angiotensin system [23]. The proposed pathophysiological processes related to unfavorable lifestyle factors, e.g., chronic inflammation and insulin resistance, are also established biological mechanisms associated with aging, a known risk factor for cancer development [24]. Moreover, a rising trend within the general population of being overweight appears to parallel a shift to the appearance of cancer at an earlier age [25]; this suggests that obesity and other unfavorable lifestyle factors not only promote tumor growth, but also affect aging and breast cancer onset. Hence, one may hypothesize that lifestyle factors play a role in relation to optimal breast cancer treatment, comorbidity, and breast cancer survival.
The main aim of the present study was, therefore, based on a population-based cohort study, with a high attendance rate and detailed medical and histopathological information, to explore the joint effect of lifestyle factors on breast cancer risk, age at onset, and survival.

\section{Materials and methods}

This population-based cohort, the Energy Balance and Breast Cancer Aspects throughout life (EBBA-Life) study $[26,27]$, is a substudy of the Troms $\varnothing$ study [28]. A total of 20,619 women, aged $>20$ years, participated in the population-based Troms $\varnothing$ study in five waves of almost identical data collection, conducted between 1986 and 2016, carried out 6-7 years apart with an attendance rate of $74.0 \%$ [28, 29]. At study entry, all participants completed questionnaire data and sampling of biological specimens, and basic clinical measurements were performed. All data collection was carried out by trained research technicians at each survey.

\section{Questionnaires}

The questionnaires were filled in at home and brought to the study site, where they were checked for completeness and inconsistency. Questionnaires included items about medical history, specific symptoms, dietary habits, lifestyle factors, reproductive factors, and use of medication including antihypertensive drugs and hormone therapy [28].

\section{Assessment of lifestyle factors and menopausal hormone therapy (MHT)}

Height and weight were measured [28], and the BMI (kg/ $\mathrm{m}^{2}$ ) was calculated. Physical activity was reported according to type of physical activity over the last 12 months (walking/cycling, recreational sport, strenuous training, or participating in sports competition), duration, and hours of intensity exercise per week. Categories of physical activity were classified as follows: (1) sedentary physical activity: reading/sitting with no participation in recreational sport activities or competitions over the last 12 months; (2) moderate physical activity: walking/cycling at least $4 \mathrm{~h}$ a week and/or minimum of $1 \mathrm{~h}$ of strenuous physical activity per week (sweating/out of breath); and (3) hard physical activity: participating in strenuous training or sports competitions regularly/several times a week and/ or exercise approximately every day. Alcohol intake was reported according to the number of days a month when alcohol was drunk, and has been validated [30]. Smoking was reported and classified as never/past smokers or current smokers. Blood pressure ( $\mathrm{mmHg}$ ) was measured [28]. 
MHT use was reported and classified as past/present use or never use. Baseline information on MHT use was obtained when the participants first entered the study, and updated MHT use was performed at each new wave between 1986 and 2016.

\section{Identification of breast cancer cases, breast tumor characteristics, and medical charts}

All breast cancer cases were identified through linkage to the Cancer Registry of Norway by using the unique, national, 11-digit identification number [31]. We obtained information on death and emigration from the Cause of Death Registry and the National Population Registry, respectively [32, 33]. Death from breast cancer was coded according to International Classification of Disease (ICD) [34]. We excluded all attendees who had a previous history of cancer, or who emigrated, died, or were diagnosed with cancer within the first year after study entry $(n=709)$. All women with missing information on BMI, physical activity, alcohol use, smoking, blood pressure, or MHT use were excluded $(n=2765)$. Thus, 17,145 women were included in the final sample. The participants were followed from the date of entry into the study until the date of breast cancer diagnosis, date of emigration, date of death, or end of follow-up (December 31, 2017), whichever event occurred first.

The breast cancer patients' medical charts were reviewed to obtain detailed clinical data, including breast cancer histological type, grade (1-3), tumor stage (1-4) according to the TNM (tumor, node, metastases) classification, and breast cancer treatment. A total of 574 women were diagnosed with incident invasive breast cancer during follow-up (Online Resource 1). Follow-up after breast cancer diagnosis was calculated from the date of the diagnosis to the date of death, emigration, or end of follow-up.

All breast tumor samples were fixed in $4 \%$ buffered formaldehyde before processing and embedding in paraffin. To obtain more complete and updated information on tumor characteristics, most $(n=407)$ of the tissue samples were analyzed on tissue microarrays (TMAs) at the University of Bergen, Norway (Centre for Cancer Biomarkers) $[26,35]$. Breast tumor specimens not reanalyzed on TMA blocks $(n=167)$ were evaluated using immunohistochemistry for hormone receptor status and Ki-67, and immunohistochemistry and fluorescence in situ hybridization for human epidermal growth factor receptor-2 (HER2). A subset of the breast cancer cases $(n=522)$ with complete information on receptor status were categorized into three molecularly defined subgroups: (1) ER positive-patients with ER-positive (with or without progesterone (PgR)-positive) and HER2-negative status; (2) HER2 positive-all patients with HER2 overexpression; and (3) triple-negative breast cancer (TNBC) - HER2-, ER--, and PgR-negative status.

\section{Statistical methods}

To characterize the change in incidence rates during follow-up, crude incidence rates were calculated as new breast cancer cases per 1000 person-years at risk for disease for the total cohort (30-90 years) and within the age groups: $\leq 55$ years and $>55$ years. Non-linearity in incidence trends was considered and an estimate calculated using a fractional polynomial in Poisson's regression models. A total of 44 models were estimated to find the best-fitting model to describe trends in breast cancer incidence.

Multivariable Cox's proportional hazard regression models were used to study whether a pre-diagnostic unfavorable lifestyle, assessed at baseline, was associated with breast cancer risk and mortality. To study the importance of the variation in lifestyle factors independently and in combination, we identified five different modifiable lifestyle-related factors associated with breast cancer development, and categorized each of them into favorable versus unfavorable based on international categorization (WCRF/AICRF, World Health Organization) [36, 37]:

1. Body composition favorable-BMI $<25 \mathrm{~kg} / \mathrm{m}^{2}$ versus unfavorable-BMI $\geq 25 \mathrm{~kg} / \mathrm{m}^{2}$.

2. Physical activity favorable-moderate or more physical activity versus unfavorable-sedentary physical activity.

3. Alcohol use favorable-no alcohol or $\leq 1$ day a month drinking alcohol versus unfavorable $->1$ day a month drinking alcohol.

4. Smoking favorable-no current smoking versus unfavorable - current smoker.

5. Hypertension favorable-systolic blood pressure $<140 \mathrm{mmHg}$, diastolic blood pressure $<90 \mathrm{mmHg}$, and no antihypertensive medication versus unfavorable-systolic blood pressure $\geq 140 \mathrm{mmHg}$ and/or diastolic blood pressure $\geq 90 \mathrm{mmHg}$, and/or use of antihypertensive medication.

The participants scored 0 for each favorable lifestyle factor and 1 for each unfavorable one, resulting in a score range of $0-5$. We then split these modifiable lifestyle factors into four categories according to the sum of unfavorable lifestyle factors: category 1 (reference): score 0 ; category 2 , score 1 ; category 3 , score 2 ; category 4 , score $3-5$. We constructed three separate regression models for each exposure to evaluate overall, premenopausal, and postmenopausal breast cancer as model-specific outcomes. Based on previous studies among women from the same cohort [27], premenopausal status was defined as age $\leq 55$ years, and all women 
aged $>55$ years were categorized as postmenopausal. MHT use is a strong risk factor for breast cancer, and therefore we ran separate time-dependent regression analyses according to updated information on MHT use during follow-up. We used a linear regression model to study the association between clustering of unfavorable lifestyle factors and age at diagnosis. One-way analysis of variance (ANOVA) was used to study the differences between number of unfavorable lifestyle factors and breast tumor characteristics.

Based on suggested biological mechanisms influencing these modifiable lifestyle factors and/or breast cancer risk and prognosis, several variables were studied as potential confounders: age (continuous), age at menarche (continuous), and number of live births (continuous). In the final analysis of survival, only age was included as a potential confounder. Breast cancer stage (categorical) did not influence our results, and was not included. A total of 111 breast cancer cases were checked for agreement between subtyping based on immunohistochemistry and TMA. We observed an agreement between these two methods in $93 \%$ of the breast cancer cases $(\kappa=0.76)$. All the tests were two sided and the statistical significance was defined by $P<0.05$. Statistical analyses calculating crude incidence of new breast cancer cases were conducted using STATA 14 (StataCorp, College Station, TX, USA). All other statistical analyses were conducted using SPSS 21.0 (IBM Corporation, Armonk, NY, USA).

\section{Results}

Among the 17,145 women included at study entry, 34.3\% were overweight, $30.7 \%$ physically inactive, and $58 \%$ consumed alcohol $>1$ day/month. We observed a $93 \%$ increase in overall age-adjusted breast cancer incidence through the period from 1995 to 2017 (incidence rate ratio 1.93, 95\% CI 1.42-2.62) (Fig. 1). Mean age at breast cancer diagnosis was 58.0 (range $31.2-92.0$ ) years, and $78.9 \%$ of the breast
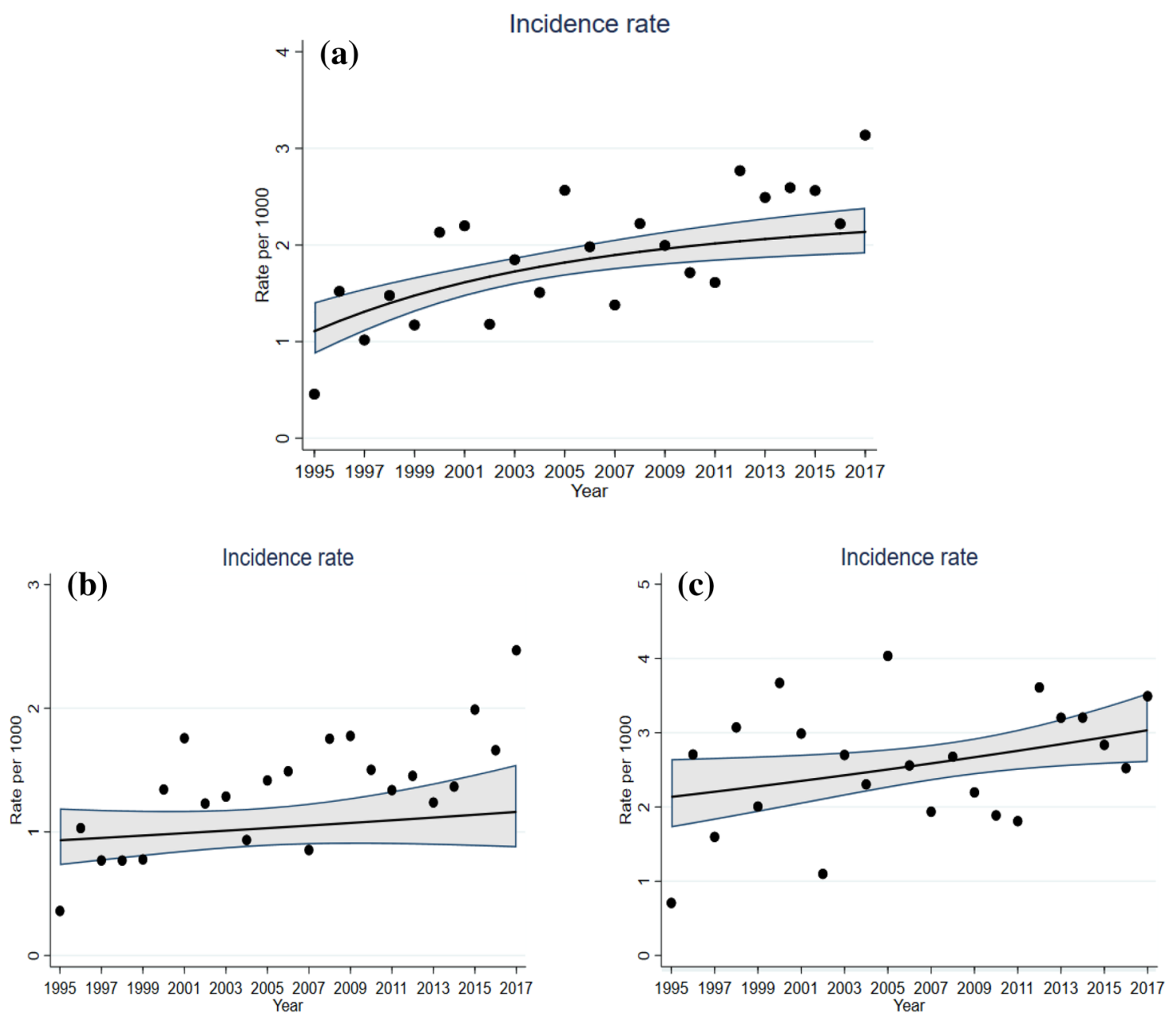

Fig. 1 Breast cancer incidence rate in the period between 1995 and 2017 among: a women aged 30-90 years, b women aged 30-55 years, and c women aged 55-90 years 
Table 1 Characteristics of women without breast cancer (non-cases) and breast cancer patients (cases): the EBBA-Life study (1986-2017)

\begin{tabular}{|c|c|c|c|}
\hline & All women $(n=17,145)^{\mathrm{a}}$ & Non-cases $(n=16571)^{\mathrm{a}}$ & $\begin{array}{l}\text { Breast cancer } \\
\text { cases }(n=574)^{\mathrm{a}}\end{array}$ \\
\hline & Mean $(\mathrm{SD}) / \%$ & Mean (SD)/\% & Mean $(\mathrm{SD}) / \%$ \\
\hline \multicolumn{4}{|l|}{ Characteristics at study entry } \\
\hline Age at attendance, years & $41.7(13.8)$ & $41.7(13.8)$ & $41.3(12.4)$ \\
\hline Follow-up, years & $20.1(10.0)$ & $20.2(11.1)$ & $16.7(7.85)$ \\
\hline Overall mortality rate, $\%$ & 14.3 & 13.9 & 26.3 \\
\hline \multicolumn{4}{|l|}{ Reproductive factors } \\
\hline Number of children & $2.11(1.36)$ & $2.11(1.36)$ & $2.03(1.26)$ \\
\hline Age at menarche, years & $13.2(1.47)$ & $13.2(1.47)$ & $13.1(1.43)$ \\
\hline \multicolumn{4}{|l|}{ Clinical variables } \\
\hline Height, $\mathrm{cm}$ & $164(6.58)$ & $164(6.57)$ & $165(6.06)$ \\
\hline BMI, $\mathrm{kg} / \mathrm{m}^{2}$ & $24.4(4.34)$ & $24.4(4.36)$ & $23.7(3.63)$ \\
\hline Systolic blood pressure, $\mathrm{mmHg}$ & $122(19,0)$ & $122(19.1)$ & $122(17.6)$ \\
\hline Diastolic blood pressure, $\mathrm{mmHg}$ & $72.4(10.9)$ & $72.3(11.0)$ & $73.3(10.7)$ \\
\hline \multicolumn{4}{|l|}{ Lifestyle factors/Comorbidity } \\
\hline MHT users ${ }^{\mathrm{b}}, \%$ & 4.5 & 4.5 & 4.2 \\
\hline Blood pressure treatment, $\%$ & 4.99 & 5.02 & 4.08 \\
\hline Overweight $^{\mathrm{c}}, \%$ & 34.3 & 34.6 & 27.1 \\
\hline Physically inactive ${ }^{\mathrm{d}}, \%$ & 30.7 & 30.6 & 32.3 \\
\hline Alcohol consumption $>1$ day/month, $\%$ & 58.1 & 58.3 & 52.7 \\
\hline Current smokers, $\%$ & 41.0 & 40.9 & 44.2 \\
\hline Hypertension $^{\mathrm{e}}, \%$ & 18.2 & 18.2 & 18.0 \\
\hline \multicolumn{4}{|l|}{ Characteristics among breast cancer cases } \\
\hline Age at diagnosis, years & & & $58.0(11.7)$ \\
\hline Observation time after diagnosis, years & & & $9.11(6.91)$ \\
\hline Cancer-specific mortality rate, $\%$ & & & 9.8 \\
\hline \multicolumn{4}{|l|}{ Tumor characteristics } \\
\hline \multicolumn{4}{|l|}{ Histological subtype, \% } \\
\hline Invasive carcinoma NST & & & 79.7 \\
\hline Invasive lobular carcinoma & & & 13.1 \\
\hline Others & & & 7.2 \\
\hline Tumor size, $\mathrm{mm}$ & & & $23.0(18.8)$ \\
\hline \multicolumn{4}{|l|}{ Stage, $\%$} \\
\hline $1-2$ & & & 82.9 \\
\hline 3 & & & 13.9 \\
\hline 4 & & & 3.2 \\
\hline \multicolumn{4}{|l|}{ Histological grading, $\%$} \\
\hline 1 & & & 28.7 \\
\hline 2 & & & 46.9 \\
\hline 3 & & & 24.4 \\
\hline Estrogen receptor positive, $\%$ & & & 78.9 \\
\hline Progesterone receptor positive, $\%$ & & & 59.8 \\
\hline HER2 positive, $\%$ & & & 16.3 \\
\hline Ki-67, \% & & & $20.6(17.8)$ \\
\hline \multicolumn{4}{|l|}{ Treatment } \\
\hline Chemotherapy, \% & & & 36.0 \\
\hline Endocrine therapy, $\%$ & & & 40.9 \\
\hline Radiation therapy, $\%$ & & & 78.5 \\
\hline
\end{tabular}

BMI Body Mass Index $\left(\mathrm{kg} / \mathrm{m}^{2}\right), H E R 2$ human epidermal growth factor receptor-2, $M H T$ menopausal hormone therapy, $N S T$ no special type, $S D$ standard deviation

${ }^{a}$ Numbers may vary due to missing information

${ }^{\mathrm{b}}$ MHT users at baseline

${ }^{\mathrm{c}}$ Overweight defined as BMI $\geq 25 \mathrm{~kg} / \mathrm{m}^{2}$

${ }^{\mathrm{d}}$ Physically inactive: reading/sitting with no participation in recreational sport activities or competitions during the last 12 months

${ }^{\text {e}}$ Systolic blood pressure $\geq 140 \mathrm{mmHg}$ and/or diastolic blood pressure $\geq 90 \mathrm{mmHg}$, and/or use of antihypertensive medication 
tumors were ER positive (Table 1). The distribution of the molecularly defined breast cancer subtypes was as follows: ER positive $(69.5 \%, n=362)$, HER 2 positive $(16.3 \%$, $n=85)$, and TNBC (14.2\%, $n=75)$ (Online Resource 2$)$.

\section{Pre-diagnostic lifestyle factors: breast cancer risk}

Among all the women, those drinking alcohol $>1$ day/month versus those drinking alcohol $0-1$ day/month had a $31 \%$ higher overall breast cancer risk (hazard ratio [HR] 1.31, 95\% CI 1.10-1.56). Current smoking versus no/previous smoking was associated with a $30 \%$ decreased premenopausal breast cancer risk (HR 0.70, 95\% CI0.53-0.91) (Table 2).

Among women who were MHT non-users, those with three to five unfavorable lifestyle factors versus those with no unfavorable lifestyle factors had more than a twofold increased risk of developing postmenopausal breast cancer (HR 2.13, 95\% CI 1.23-3.69) (Table 2). No clear association was observed between clustering of unfavorable lifestyle factors and premenopausal breast cancer risk (Table 2).

After stratification by breast cancer molecular subtype, women with three to five unfavorable lifestyle factors versus no unfavorable lifestyle factors had a $43 \%$ increased
ER-positive breast cancer risk (HR 1.43, 95\% CI 0.97-2.10, $P_{\text {trend }}=0.096$ ). Among postmenopausal women who were MHT non-users, those with three to five unfavorable lifestyle factors versus no unfavorable lifestyle factors had a $142 \%$ increased risk for ER-positive postmenopausal breast cancer (HR 2.42, 95\% CI 1.27-4.63) (Online Resource 3). No association was observed between clustering of unfavorable lifestyle factors and HER2-positive cancer and TNBC overall (Online Resource 2).

\section{Pre-diagnostic lifestyle factors: age at diagnosis}

Among MHT non-users who developed postmenopausal breast cancer, women with three to five unfavorable lifestyle factors were 3.4 years younger at diagnosis than those with no unfavorable lifestyle factors (64.8 years versus 68.2 years, $P=0.032$ ) (Table 3 ). Furthermore, these MHT non-users with three to five unfavorable lifestyle factors had larger tumors than those with no unfavorable lifestyle factors (26.3 versus $12.3 \mathrm{~mm}, P=0.023$ ). Other tumor characteristics were not statistically differently distributed throughout

Table 2 Age-adjusted hazard ratios for incident breast cancer overall and according to menopausal status and MHT use

\begin{tabular}{|c|c|c|c|c|c|c|c|c|}
\hline & \multirow{2}{*}{\multicolumn{2}{|c|}{ All breast cancers }} & \multirow{2}{*}{\multicolumn{2}{|c|}{ Premenopausal breast cancer }} & \multicolumn{4}{|c|}{ Postmenopausal breast cancer } \\
\hline & & & & & \multicolumn{2}{|c|}{ MHT non-users } & \multicolumn{2}{|c|}{ MHT users } \\
\hline & $n=574$ & HR $(95 \% \mathrm{CI})$ & $n=246$ & HR $(95 \%$ CI $)$ & $n=237$ & HR $(95 \% \mathrm{CI})$ & $n=91$ & HR $(95 \%$ CI $)$ \\
\hline \multicolumn{9}{|c|}{ Modifiable lifestyle factors } \\
\hline Overweight $^{\mathrm{a}}$ & & $0.92(0.75-1.12)$ & & $0.88(0.64-1.23)$ & & $1.16(0.86-1.55)$ & & $0.88(0.53-1.33)$ \\
\hline Physical inactivity ${ }^{\mathrm{b}}$ & & $1.07(0.90-1.29)$ & & $1.20(0.90-1.59)$ & & $1.10(0.81-1.48)$ & & $0.79(0.51-1.23)$ \\
\hline Alcohol use ${ }^{c}$ & & $1.31(1.10-1.56)$ & & $1.05(0.80-1.38)$ & & $1.57(1.20-2.06)$ & & $1.18(0.82-1.71)$ \\
\hline Cigarette smoking $^{\mathrm{d}}$ & & $0.94(0.79-1.12)$ & & $0.70(0.53-0.91)$ & & $1.16(0.89-1.59)$ & & $1.11(0.77-1.16)$ \\
\hline Hypertension $^{\mathrm{e}}$ & & $0.97(0.67-1.13)$ & & $1.17(0.72-1.90)$ & & $0.85(0.60-1.21)$ & & $1.00(0.60-1.68)$ \\
\hline \multicolumn{9}{|c|}{ Number of unfavorable lifestyle factors } \\
\hline 0 (ref) & 62 & 1.00 & 34 & 1.00 & 17 & 1.00 & 11 & 1.00 \\
\hline 1 & 200 & $1.41(1.04-1.78)$ & 91 & $1.05(0.71-1.55)$ & 71 & $1.66(0.97-2.83)$ & 38 & $1.58(0.86-2.92)$ \\
\hline 2 & 176 & $1.26(0.93-1.71)$ & 79 & $0.90(0.60-1.34)$ & 72 & $1.72(1.01-2.93)$ & 26 & $1.29(0.67-2.45)$ \\
\hline $3-5$ & 136 & $1.34(0.97-1.85)$ & 42 & $0.83(0.53-1.31)$ & 77 & $2.13(1.23-3.69)$ & 17 & $1.22(0.60-2.52)$ \\
\hline$P_{\text {trend }}$ & & 0.348 & & 0.222 & & 0.011 & & 0.999 \\
\hline
\end{tabular}

Cox proportional hazard model

Adjusted to age, age at menarche, and number of live births

$C I$ confidence interval, $H R$ Hazard ratio, $M H T$ menopausal hormone therapy, $n$ number of cases, $r e f$ reference

${ }^{a}$ Overweight defined by BMI $\geq 25 \mathrm{~kg} / \mathrm{m}^{2}$

${ }^{b}$ Physical inactivity defined by reading/sitting with no participation in recreational sport activities or competitions during last 12 months

${ }^{c}$ Alcohol use defined by $>1$ day of alcohol use per month

${ }^{\mathrm{d}}$ Current cigarette smoking

${ }^{\mathrm{e}}$ Hypertension defined by systolic blood pressure $>140 \mathrm{mmHg}$ and/or diastolic blood pressure $>90 \mathrm{mmHg}$, and/or use of antihypertensive medication 
Table 3 Age at diagnosis among breast cancer patients according to number of unfavorable lifestyle factors by menopausal status at diagnosis and MHT use

\begin{tabular}{|c|c|c|c|c|c|c|c|c|}
\hline & \multirow{2}{*}{\multicolumn{2}{|c|}{ All breast cancers }} & \multirow{2}{*}{\multicolumn{2}{|c|}{ Premenopausal breast cancer }} & \multicolumn{4}{|c|}{ Postmenopausal breast cancer } \\
\hline & & & & & \multicolumn{2}{|c|}{ MHT non-users } & \multicolumn{2}{|c|}{ MHT users } \\
\hline & $n=574$ & Age at diagnosis (SE) & $n=246$ & Age at diagnosis (SE) & $n=237$ & Age at diagnosis (SE) & $n=91$ & Age at diagnosis (SE) \\
\hline \multicolumn{9}{|c|}{ Number of unfavorable lifestyle factors ${ }^{a}$} \\
\hline 0 & 62 & $58.8(0.97)$ & 34 & $49.2(0.80)$ & 17 & $68.2(1.37)$ & 11 & $63.0(1.53)$ \\
\hline 1 & 200 & $58.5(0.54)$ & 91 & $47.5(0.48)$ & 71 & $66.7(0.68)$ & 38 & $65.1(0.81)$ \\
\hline 2 & 176 & $57.4(0.57)$ & 79 & $46.6(0.52)$ & 72 & $65.7(0.67)$ & 26 & $66.0(0.97)$ \\
\hline $3-5$ & 136 & $58.0(0.67)$ & 42 & $48.6(0.72)$ & 77 & $64.8(0.68)$ & 17 & $65.6(1.23)$ \\
\hline$P^{\mathrm{b}}$ & & 0.517 & & 0.585 & & 0.032 & & 0.198 \\
\hline
\end{tabular}

Linear regression model

Covariates: Age at study entry

$C I$ confidence interval, $M H T$ menopausal hormone therapy, $n$ number of cases, $S E$ standard error

${ }^{a}$ Unfavorable lifestyle factors: overweight, physical inactivity, alcohol use, smoking, and hypertension

${ }^{\mathrm{b}} p$ value reflecting difference between no unfavorable lifestyle factors and three to five unfavorable lifestyle factors

the four categories of unfavorable lifestyle factors (Online Resource 4).

\section{Pre-diagnostic lifestyle factors: overall mortality and breast cancer-specific mortality}

Among all breast cancer cases combined, we observed a 96\% increased overall mortality risk for those breast cancer cases with three to five unfavorable lifestyle factors versus no unfavorable lifestyle factors (HR 1.96, 95\% CI 1.01-3.80) (Table 4, Fig. 2). A positive trend between clustering of unfavorable lifestyle factors and overall mortality was observed among premenopausal breast cancer patients $\left(P_{\text {trend }}=0.020\right)$ (Table 4).
Among postmenopausal women, we observed a suggestively inverse trend between clustering of unfavorable lifestyle factors and breast cancer mortality $\left(P_{\text {trend }}=0.050\right)($ Table 4$)$.

\section{Discussion}

In this population-based cohort we observed, over 20 years of follow-up, a 93\% increased breast cancer incidence. Furthermore, among women who had a clustering of prediagnostic unfavorable lifestyle factors, compared with women who had a favorable lifestyle, we demonstrated a twofold increase in postmenopausal breast cancer risk.

Table 4 Age-adjusted hazards ratios between lifestyle and overall and breast cancer mortality by menopausal status

\begin{tabular}{|c|c|c|c|c|c|c|c|c|c|}
\hline & \multicolumn{3}{|c|}{ All breast cancer cases $(n=574)$} & \multicolumn{3}{|c|}{ Premenopausal breast cancer $(n=245)$} & \multicolumn{3}{|c|}{ Postmenopausal breast cancer $(n=329)$} \\
\hline & Cases $(n)$ & $\begin{array}{l}\text { Overall } \\
\text { mortality (150 } \\
\text { deaths) }\end{array}$ & $\begin{array}{l}\text { Breast cancer } \\
\text { mortality ( } 56 \\
\text { deaths) }\end{array}$ & Cases $(n)$ & $\begin{array}{l}\text { Overall } \\
\text { mortality (55 } \\
\text { deaths) }\end{array}$ & $\begin{array}{l}\text { Breast cancer } \\
\text { mortality ( } 26 \\
\text { deaths) }\end{array}$ & Cases $(n)$ & $\begin{array}{l}\text { Overall } \\
\text { mortality (95 } \\
\text { deaths) }\end{array}$ & $\begin{array}{l}\text { Breast cancer } \\
\text { mortality ( } 30 \\
\text { deaths) }\end{array}$ \\
\hline & & $\operatorname{HR}(95 \% \mathrm{CI})$ & $\operatorname{HR}(95 \% \mathrm{CI})$ & & HR $(95 \%$ CI $)$ & HR $(95 \%$ CI $)$ & & $\operatorname{HR}(95 \% \mathrm{CI})$ & $\operatorname{HR}(95 \% \mathrm{CI})$ \\
\hline \multicolumn{10}{|c|}{ Number of unfavorable lifestyle factors } \\
\hline 0 (ref) & 62 & 1.00 & 1.00 & 34 & 1.00 & 1.00 & 28 & 1.00 & 1.00 \\
\hline 1 & 200 & $\begin{array}{l}1.10(0.57- \\
2.15)\end{array}$ & $\begin{array}{c}1.14(0.46- \\
2.82)\end{array}$ & 91 & $\begin{array}{l}0.94(0.34- \\
2.59)\end{array}$ & $\begin{array}{l}0.90(0.23- \\
3.40)\end{array}$ & 109 & $\begin{array}{l}1.28(0.53- \\
3.13)\end{array}$ & $1.37(0.39-4.79)$ \\
\hline 2 & 176 & $\begin{array}{l}1.45(0.75- \\
2.82)\end{array}$ & $\begin{array}{l}0.97(0-37- \\
2.49)\end{array}$ & 79 & $\begin{array}{l}1.71(0.63- \\
4.61)\end{array}$ & $\begin{array}{l}1.21(0.32- \\
4.62)\end{array}$ & 98 & $\begin{array}{l}1.08(0.44- \\
2.70)\end{array}$ & $\begin{array}{l}0.56(0.14- \\
2.26)\end{array}$ \\
\hline $3-5$ & 136 & $\begin{array}{l}1.96(1.01- \\
3.80)\end{array}$ & $\begin{array}{l}1.05(0.39- \\
2.81)\end{array}$ & 42 & $\begin{array}{l}2.16(0.77- \\
6.06)\end{array}$ & $\begin{array}{l}1.78(0.44- \\
7.12)\end{array}$ & 94 & $\begin{array}{l}1.39(0.57- \\
3.39)\end{array}$ & $\begin{array}{c}0.47(0.11- \\
1.96)\end{array}$ \\
\hline$P_{\text {trend }}$ & & 0.005 & 0.874 & & 0.020 & 0.260 & & 0.553 & 0.050 \\
\hline
\end{tabular}

Multivariable Cox proportional hazard regression models

Adjusted for age (continuous)

$C I$ confidence interval, $H R$ Hazard ratio, $n$ number of cases, $r e f$ reference 
Fig. 2 Women diagnosed with breast cancer in the EBBA-Life study $(n=574)$. Number of unfavorable lifestyle factors and a overall mortality, and $\mathbf{b}$ breast cancer mortality Adjusted for age

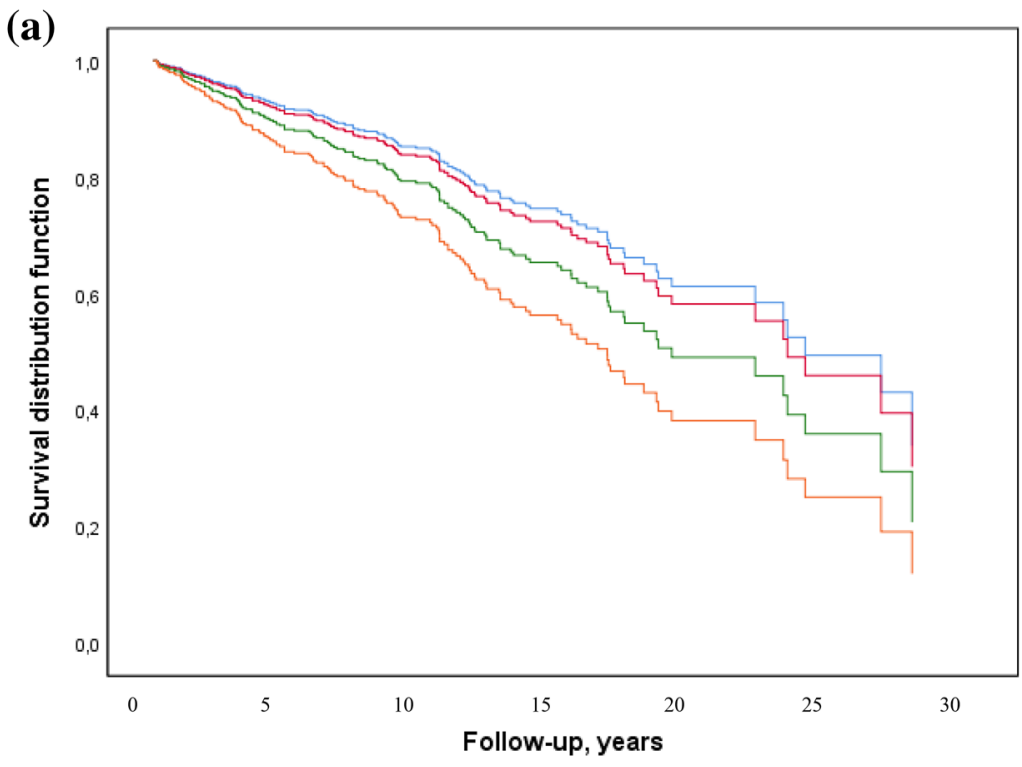

Number of unfavorable lifestyle factors

$$
\begin{array}{ll}
\neg 0 \\
\neg 1 \\
\neg 2 \\
-\neg 3-5
\end{array}
$$

(b)

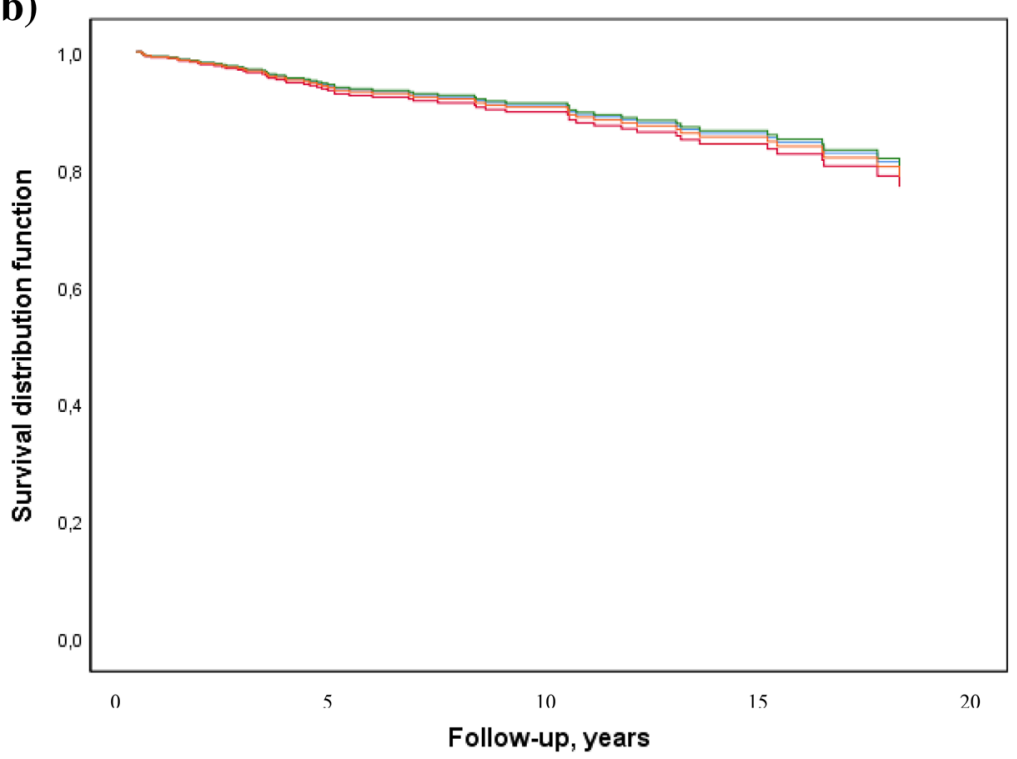

Number of unfavorable lifestyle factors

$\neg 0$

$\neg 1$

$\neg 2$

$\neg 3-5$
This increased breast cancer risk was restricted to MHT non-users, and confined to the most common breast cancer subtype: ER-positive breast tumors. Moreover, postmenopausal women with a pre-diagnostic unfavorable lifestyle were 3.4 years younger at breast cancer diagnosis compared with women who had a favorable lifestyle. Our study also demonstrated that a pre-diagnostic unfavorable lifestyle was associated with almost a two times higher overall mortality risk among women with breast cancer.

Our observed results extend previous studies, but are also in part supported by others $[38,39]$. The steady increase in breast cancer incidence over the last three decades observed in our cohort is in line with findings in comparable populations [1]. Of note, an increased breast cancer incidence observed in the present study is unlikely to have been influenced by any breast cancer-screening program introduced many years ahead [40].

Our findings of a dose-response association between a joint combination of lifestyle factors and breast cancer risk with a twofold increase in postmenopausal breast cancer risk among women with an unfavorable lifestyle are supported [41-44]. In a large prospective study including mainly postmenopausal women [45], adherence to dietary guidelines [46] was associated with a decreased breast cancer risk, in particular ER-positive breast cancer tumors. In the Nurse's Health Study cohort, a decreased postmenopausal breast cancer risk was observed, especially for ER-positive breast cancer, among women with the lowest weight gain, no alcohol consumption, high physical activity level, and had no MHT use [47]. Moreover, postmenopausal women 
who participated in the Norwegian Breast Cancer Screening Program, self-reported lifestyle factors were associated with increased risk for ER-positive luminal A-like and luminal B-like HER2-positive breast cancer [48]. Of note, MHT use has been documented as a strong independent risk factor for breast cancer development [49]. This underlines the importance of investigating the clustering of modifiable lifestyle factors stratified by MHT users and non-users [49-51]. However, the well-defined interaction between obesity, physical inactivity, and breast cancer among MHT non-users and not among MHT users is not generally present for alcohol [52] and smoking [53]. It is of interest that, in a recent study among women participating in the UK Biobank study, a healthy lifestyle, including diet, physical activity, smoking, alcohol intake, and body composition, was observed to attenuate the impact of genetic factors on invasive breast cancer risk [54]. In our age-adjusted regression analysis presenting the lifestyle factors, smoking was inversely related to premenopausal breast cancer risk, in contrast to other studies [55]. Our observation may be a chance finding, and based on recent observations and potential biological mechanisms operating, we chose to include smoking as a lifestyle-associated breast cancer risk factor.

To our knowledge, our results showing an association between an unfavorable lifestyle and earlier age at diagnosis of sporadic breast cancer have not previously been reported in a population-based cohort study. However, in a recent study weight gain was associated with earlier age at diagnosis [56]. Moreover, one early study showed that among BRCA1/BRCA2 mutations carriers, physical exercise and healthy weight were associated with delayed age at breast cancer onset [57]. Hence, previous results and our results suggest that an unfavorable lifestyle may promote tumor growth and alter breast cancer onset in both BRCAI/II mutation carriers and in those at risk of sporadic breast cancer.

In our study, we observed that a pre-diagnostic unfavorable lifestyle was associated with an almost two times higher overall mortality. An association between obesity and more advanced stage and higher grade at diagnosis among postmenopausal breast cancer patients has been found [58]. We observed that women with postmenopausal breast cancer who were MHT non-users and had an unfavorable lifestyle, including being overweight, were likely to have larger tumors compared with women with a favorable lifestyle. However, larger tumor size at diagnosis among postmenopausal breast cancer patients was not translated into shortened breast cancer-specific survival in our study. The association between an overall unfavorable lifestyle and higher overall mortality risk may reflect that an unfavorable lifestyle is often associated with other comorbid conditions [59, 60]. Consequently, breast cancer patients, even if they are cured of their cancer, may experience a reduced overall life expectancy due to pre-existing susceptibility to comorbidities and late adverse effects of treatment [61, 62]. This may, in particular, play a role for young breast cancer patients, as they tend to present with more aggressive tumor characteristics [63], which requires more comprehensive treatment, putting these women at risk of developing more severe treatment-induced chronic adverse effects [64]. Hence, the potential long period of post-diagnostic survival for most breast cancer patients provides a context in which long-term exposure to an unfavorable lifestyle may have a substantial impact on morbidity and overall mortality. Of note, we observed, among women with postmenopausal breast cancer, an inverse association between pre-diagnostic unfavorable lifestyle and breast cancer mortality. This observation may reflect that postmenopausal women with an unfavorable lifestyle are prone to die of causes other than their breast cancer [65].

The exact biological mechanisms explaining the association between lifestyle and breast cancer development have yet to be established, but an unfavorable lifestyle may accelerate aging and increase tumor growth, and result in chronic inflammation, metabolic dysfunction, and DNA methylation [66-68]. Telomere length has been proposed as a biomarker of biological age and a risk factor for cancer [69]. The unfavorable lifestyle factors included in our study have been independently observed to shorten telomere length, leading to accelerated aging [70-73], and potentially influence breast cancer prognosis [74]. An association between telomere length and breast cancer risk has shown conflicting results $[75,76]$. However, one may propose that a healthy lifestyle may reduce the pace of telomere shortening, and delay the onset of age-related diseases, including breast cancer. In addition, high elevated levels of advanced glycation end products, observed in ER-positive breast cancers, have been associated with physical inactivity, tumor growth, and therapy resistance [77].

Our study had several strengths, which include its population-based approach, high attendance rate, the use of a standardized protocol for data collection, and measured height and weight. The study had high completeness rates of identification of breast cancer cases (Cancer Registry of Norway), and identification of death and emigration (Cause of death Registry). In an evaluation of the data quality of the Cancer Registry of Norway, completeness of reporting was estimated to be very high, $98.8 \%$ [31], limiting the possibility for any misclassification related to identification of cases. Thus, it is less likely that loss to follow-up or inadequate reporting influences our risk estimate, further reducing the chance of biased observations. Moreover, to lessen the chance that undiagnosed cancer could influence our results, we limited our study population to women who had no history of previous breast or other cancer within the first year after study inclusion. All medical records for the breast cancer patients were 
carefully reviewed by trained physicians, and included systematic abstraction of histopathology, oncological treatment, and recurrence and death [27]. The study also has several limitations. We did not have data on age at first pregnancy, family history of disease, weight at young adulthood, and adult weight gain. Moreover, the lifestyle factors and other baseline variables, except menopausal hormone therapy use, were based on a single pre-diagnostic measure collected average 20.1 years before censored. Thus, we were therefore unable to account for longitudinal changes in these factors on breast cancer risk and survival. However, tracking studies have shown that women tend to follow a trajectory of BMI, physical activity, blood pressure, and alcohol intake [78-80], suggesting an accumulated lifetime exposure. The sample size narrowed our possibility of performing further stratified analysis by breast cancer subtypes.

In conclusion, our study supports a dose-response association between lifestyle behavior and sporadic breast cancer. An unfavorable lifestyle may alter biological pathways, accelerating tumor growth, and thereby increase breast cancer risk, lower the age at onset of sporadic breast cancer, and shorten the life expectancy among breast cancer patients. Consequently, our results not only support a key role of a healthy lifestyle to improve breast cancer prevention, but also suggest that tailored personal lifestyle advice should be included in daily clinical practice of potential importance for treatment outcomes and life expectancy for women diagnosed with breast cancer.

Acknowledgements Open Access funding provided by University of Oslo (incl Oslo University Hospital). We thank the participants in the municipality of Troms $\varnothing$ Northern Norway, The Institute of Community Medicine (University of Troms $\varnothing$ ), The Department of Oncology (University Hospital of Northern Norway), The Department of Pathology (Haukeland University Hospital, Bergen, Norway), and The Department of Oncology of Oslo University Hospital. The study has used data from the Cancer Registry of Norway; the interpretation and reporting of these data are the sole responsibility of the authors, and no endorsement by the Cancer Registry of Norway is intended nor should be inferred.

Funding This study was funded by The Norwegian Extra Foundation for Health and Rehabilitation (2013/FOM 5628) and the Norwegian Cancer Society (2012/2/0054). Dr. McTiernan was supported by the Breast Cancer Research Foundation (BCRF-18-107 and BCRF-19-107).

\section{Compliance with ethical standards}

Conflict of interest All authors declare that they have no conflict of interest.

Ethical approval The EBBA-Life study has been approved by the Regional Committee for Medical and Health Research Ethics (2015/599). All procedures performed in studies involving human participants were in accordance with the ethical standards of the insti- tutional and/or national research committee and with the 1964 Helsinki declaration and its later amendments or comparable ethical standards.

Informed consent Informed consent was obtained from all individual participants included in the study.

Open Access This article is licensed under a Creative Commons Attribution 4.0 International License, which permits use, sharing, adaptation, distribution and reproduction in any medium or format, as long as you give appropriate credit to the original author(s) and the source, provide a link to the Creative Commons licence, and indicate if changes were made. The images or other third party material in this article are included in the article's Creative Commons licence, unless indicated otherwise in a credit line to the material. If material is not included in the article's Creative Commons licence and your intended use is not permitted by statutory regulation or exceeds the permitted use, you will need to obtain permission directly from the copyright holder. To view a copy of this licence, visit http://creativecommons.org/licenses/by/4.0/.

\section{References}

1. Harvie M, Howell A, Evans DG (2015) Can diet and lifestyle prevent breast cancer: what is the evidence? American Society of Clinical Oncology educational book/ASCO. Am Soc Clin Oncol. https://doi.org/10.14694/EdBook_AM.2015.35.e66

2. Anderson WF, Rosenberg PS, Petito L, Katki HA, Ejlertsen B, Ewertz M, Rasmussen BB, Jensen MB, Kroman N (2013) Divergent estrogen receptor-positive and -negative breast cancer trends and etiologic heterogeneity in Denmark. Int J Cancer 133:22012206. https://doi.org/10.1002/ijc.28222

3. Capocaccia R, Gatta G, Dal Maso L (2015) Life expectancy of colon, breast, and testicular cancer patients: an analysis of USSEER population-based data. Ann Oncol 26:1263-1268. https:// doi.org/10.1093/annonc/mdv131

4. Han X, Stevens J, Truesdale KP, Bradshaw PT, KucharskaNewton A, Prizment AE, Platz EA, Joshu CE (2014) Body mass index at early adulthood, subsequent weight change and cancer incidence and mortality. Int J Cancer 135:2900-2909. https://doi. org/10.1002/ijc. 28930

5. Key TJ, Appleby PN, Reeves GK, Roddam A, Dorgan JF, Longcope C, Stanczyk FZ, Stephenson HE Jr, Falk RT, Miller R, Schatzkin A, Allen DS, Fentiman IS, Key TJ, Wang DY, Dowsett M, Thomas HV, Hankinson SE, Toniolo P, Akhmedkhanov A, Koenig K, Shore RE, Zeleniuch-Jacquotte A, Berrino F, Muti P, Micheli A, Krogh V, Sieri S, Pala V, Venturelli E, Secreto G, Barrett-Connor E, Laughlin GA, Kabuto M, Akiba S, Stevens RG, Neriishi K, Land CE, Cauley JA, Kuller LH, Cummings SR, Helzlsouer KJ, Alberg AJ, Bush TL, Comstock GW, Gordon GB, Miller SR, Longcope C (2003) Body mass index, serum sex hormones, and breast cancer risk in postmenopausal women. J Natl Cancer Inst 95:1218-1226

6. Chan DSM, Abar L, Cariolou M, Nanu N, Greenwood DC, Bandera EV, McTiernan A, Norat T (2019) World Cancer Research Fund International: continuous update project-systematic literature review and meta-analysis of observational cohort studies on physical activity, sedentary behavior, adiposity, and weight change and breast cancer risk. Cancer Causes Control 30:1183-1200. https://doi.org/10.1007/s10552-019-01223-w

7. Chan DS, Vieira AR, Aune D, Bandera EV, Greenwood DC, McTiernan A, Navarro Rosenblatt D, Thune I, Vieira R, Norat T (2014) Body mass index and survival in women with breast cancer-systematic literature review and meta-analysis of 82 follow-up 
studies. Ann Oncol 25:1901-1914. https://doi.org/10.1093/annon c/mdu042

8. Thune I, Brenn T, Lund E, Gaard M (1997) Physical activity and the risk of breast cancer. N Engl J Med 336:1269-1275. https:// doi.org/10.1056/nejm199705013361801

9. McTiernan A, Friedenreich CM, Katzmarzyk PT, Powell KE, Macko R, Buchner D, Pescatello LS, Bloodgood B, Tennant B, Vaux-Bjerke A, George SM, Troiano RP, Piercy KL (2019) Physical activity in cancer prevention and survival: a systematic review. Med Sci Sports Exerc 51:1252-1261. https://doi.org/10.1249/ mss.0000000000001937

10. Shield KD, Soerjomataram I, Rehm J (2016) Alcohol use and breast cancer: a critical review. Alcohol Clin Exp Res 40:11661181. https://doi.org/10.1111/acer.13071

11. Hamajima N, Hirose K, Tajima K, Rohan T, Calle EE, Heath CW Jr, Coates RJ, Liff JM, Talamini R, Chantarakul N, Koetsawang S, Rachawat D, Morabia A, Schuman L, Stewart W, Szklo M, Bain C, Schofield F, Siskind V, Band P, Coldman AJ, Gallagher RP, Hislop TG, Yang P, Kolonel LM, Nomura AM, Hu J, Johnson KC, Mao Y, De Sanjose S, Lee N, Marchbanks P, Ory HW, Peterson HB, Wilson HG, Wingo PA, Ebeling K, Kunde D, Nishan P, Hopper JL, Colditz G, Gajalanski V, Martin N, Pardthaisong T, Silpisornkosol S, Theetranont C, Boosiri B, Chutivongse S, Jimakorn P, Virutamasen P, Wongsrichanalai C, Ewertz M, Adami HO, Bergkvist L, Magnusson C, Persson I, Chang-Claude J, Paul C, Skegg DC, Spears GF, Boyle P, Evstifeeva T, Daling JR, Hutchinson WB, Malone K, Noonan EA, Stanford JL, Thomas DB, Weiss NS, White E, Andrieu N, Bremond A, Clavel F, Gairard B, Lansac J, Piana L, Renaud R, Izquierdo A, Viladiu P, Cuevas HR, Ontiveros P, Palet A, Salazar SB, Aristizabel N, Cuadros A, Tryggvadottir L, Tulinius H, Bachelot A, Le MG, Peto J, Franceschi S, Lubin F, Modan B, Ron E, Wax Y, Friedman GD, Hiatt RA, Levi F, Bishop T, Kosmelj K et al (2002) Alcohol, tobacco and breast cancer-collaborative reanalysis of individual data from 53 epidemiological studies, including 58,515 women with breast cancer and 95,067 women without the disease. Br J Cancer 87:1234-1245. https://doi.org/10.1038/sj.bjc.6600596

12. Frydenberg H, Flote VG, Larsson IM, Barrett ES, Furberg AS, Ursin G, Wilsgaard T, Ellison PT, McTiernan A, Hjartaker A, Jasienska G, Thune I (2015) Alcohol consumption, endogenous estrogen and mammographic density among premenopausal women. Breast Cancer Res 17:103. https://doi.org/10.1186/s1305 8-015-0620-1

13. Chen WY, Rosner B, Hankinson SE, Colditz GA, Willett WC (2011) Moderate alcohol consumption during adult life, drinking patterns, and breast cancer risk. JAMA 306:1884-1890. https:// doi.org/10.1001/jama.2011.1590

14. Jung S, Wang M, Anderson K, Baglietto L, Bergkvist L, Bernstein L, van den Brandt PA, Brinton L, Buring JE, Eliassen AH, Falk R, Gapstur SM, Giles GG, Goodman G, Hoffman-Bolton J, Horn-Ross PL, Inoue M, Kolonel LN, Krogh V, Lof M, Maas P, Miller AB, Neuhouser ML, Park Y, Robien K, Rohan TE, Scarmo S, Schouten LJ, Sieri S, Stevens VL, Tsugane S, Visvanathan K, Wilkens LR, Wolk A, Weiderpass E, Willett WC, ZeleniuchJacquotte A, Zhang SM, Zhang X, Ziegler RG, Smith-Warner SA (2016) Alcohol consumption and breast cancer risk by estrogen receptor status: in a pooled analysis of 20 studies. Int J Epidemiol 45:916-928. https://doi.org/10.1093/ije/dyv156

15. Seretis A, Cividini S (2019) Association between blood pressure and risk of cancer development: a systematic review and metaanalysis of observational studies. Sci Rep 9:8565. https://doi. org/10.1038/s41598-019-45014-4

16. Han H, Guo W, Shi W, Yu Y, Zhang Y, Ye X, He J (2017) Hypertension and breast cancer risk: a systematic review and metaanalysis. Sci Rep 7:44877. https://doi.org/10.1038/srep44877
17. Dibaba DT, Ogunsina K, Braithwaite D, Akinyemiju T (2019) Metabolic syndrome and risk of breast cancer mortality by menopause, obesity, and subtype. Breast Cancer Res Treat 174:209218. https://doi.org/10.1007/s10549-018-5056-8

18. Johnson KC, Miller AB, Collishaw NE, Palmer JR, Hammond SK, Salmon AG, Cantor KP, Miller MD, Boyd NF, Millar J, Turcotte F (2011) Active smoking and secondhand smoke increase breast cancer risk: the report of the Canadian Expert Panel on tobacco smoke and breast cancer risk (2009). Tobacco Control 20:e2. https ://doi.org/10.1136/tc.2010.035931

19. Gaudet MM, Carter BD, Brinton LA, Falk RT, Gram IT, Luo J, Milne RL, Nyante SJ, Weiderpass E, Beane Freeman LE, Sandler DP, Robien K, Anderson KE, Giles GG, Chen WY, Feskanich D, Braaten T, Isaacs C, Butler LM, Koh WP, Wolk A, Adami HO, White E, Margolis KL, Thun MJ, Gapstur SM (2017) Pooled analysis of active cigarette smoking and invasive breast cancer risk in 14 cohort studies. Int J Epidemiol 46:881-893. https://doi. org/10.1093/ije/dyw288

20. Gilbert CA, Slingerland JM (2013) Cytokines, obesity, and cancer: new insights on mechanisms linking obesity to cancer risk and progression. Annu Rev Med 64:45-57. https://doi. org/10.1146/annurev-med-121211-091527

21. Howe LR, Subbaramaiah K, Hudis CA, Dannenberg AJ (2013) Molecular pathways: adipose inflammation as a mediator of obesity-associated cancer. Clin Cancer Res 19:6074-6083. https ://doi.org/10.1158/1078-0432.ccr-12-2603

22. Koene RJ, Prizment AE, Blaes A, Konety SH (2016) Shared risk factors in cardiovascular disease and cancer. Circulation 133:1104-1114. https://doi.org/10.1161/circulatio naha.115.020406

23. Rasha F, Ramalingam L, Gollahon L, Rahman RL, Rahman SM, Menikdiwela K, Moustaid-Moussa N (2019) Mechanisms linking the renin-angiotensin system, obesity, and breast cancer. Endocr Relat Cancer 26:R653-r672. https://doi.org/10.1530/ erc-19-0314

24. Epel ES (2009) Psychological and metabolic stress: a recipe for accelerated cellular aging? Hormones (Athens, Greece) 8:7-22. https://doi.org/10.14310/horm.2002.1217

25. Berger NA (2018) Young Adult Cancer: Influence of the Obesity Pandemic. Obesity (Silver Spring, Md.) 26:641-650. https://doi. org/10.1002/oby.22137

26. Lofterod T, Mortensen ES, Nalwoga H, Wilsgaard T, Frydenberg H, Risberg T, Eggen AE, McTiernan A, Aziz S, Wist EA, Stensvold A, Reitan JB, Akslen LA, Thune I (2018) Impact of pre-diagnostic triglycerides and HDL-cholesterol on breast cancer recurrence and survival by breast cancer subtypes. BMC Cancer 18:654. https://doi.org/10.1186/s12885-018-4568-2

27. Frydenberg H, Thune I, Lofterod T, Mortensen ES, Eggen AE, Risberg T, Wist EA, Flote VG, Furberg AS, Wilsgaard T, Akslen LA, McTiernan A (2016) Pre-diagnostic high-sensitive C-reactive protein and breast cancer risk, recurrence, and survival. Breast Cancer Res Treat. https://doi.org/10.1007/s10549-015-3671-1

28. Eggen AE, Mathiesen EB, Wilsgaard T, Jacobsen BK, Njolstad I (2013) The sixth survey of the Tromso Study (Tromso 6) in 2007-08: collaborative research in the interface between clinical medicine and epidemiology: study objectives, design, data collection procedures, and attendance in a multipurpose populationbased health survey. Scand J Public Health 41:65-80. https://doi. org/10.1177/1403494812469851

29. Jacobsen BK, Eggen AE, Mathiesen EB, Wilsgaard T, Njolstad I (2012) Cohort profile: the Tromso Study. Int J Epidemiol 41:961967. https://doi.org/10.1093/ije/dyr049

30. Rognmo K, Bergvik S, Rosenvinge JH, Bratlid KL, Friborg O (2019) Gender differences in the bidirectional relationship between alcohol consumption and sleeplessness: the Tromso 
study. BMC Public Health 19:444. https://doi.org/10.1186/s1288 9-019-6801-6

31. Larsen IK, Smastuen M, Johannesen TB, Langmark F, Parkin DM, Bray F, Moller B (2009) Data quality at the Cancer Registry of Norway: an overview of comparability, completeness, validity and timeliness. Eur J Cancer (Oxford, England: 1990) 45:1218-1231. https://doi.org/10.1016/j.ejca.2008.10.037

32. Statistics Norway (2018) Norwegian cause of death register. https ://ssb.no/a/english/kortnavn/dodsarsak_en/about.html

33. The Norwegian Tax Administation (2018) The Norwegian national registry. https://www.skatteetaten.no/en/person/natio nal-registry/

34. Organization WH (2018) Classification of diseases. https://www. who.int/classifications/icd/en/

35. Knutsvik G, Stefansson IM, Aziz S, Arnes J, Eide J, Collett K, Akslen LA (2014) Evaluation of Ki67 expression across distinct categories of breast cancer specimens: a population-based study of matched surgical specimens, core needle biopsies and tissue microarrays. PLoS ONE 9:e112121. https://doi.org/10.1371/journ al.pone. 0112121

36. Fund WCR Cancer Prevention Recommendations (2018). https:// www.wcrf.org/dietandcancer/cancer-prevention-recommendations

37. Organization WH Hypertension (2019). https://www.who.int/healt h-topics/hypertension/

38. McKenzie F, Ferrari P, Freisling H, Chajes V, Rinaldi S, de Batlle J, Dahm CC, Overvad K, Baglietto L, Dartois L, Dossus L, Lagiou P, Trichopoulos D, Trichopoulou A, Krogh V, Panico S, Tumino R, Rosso S, Bueno-de-Mesquita HB, May A, Peeters PH, Weiderpass E, Buckland G, Sanchez MJ, Navarro C, Ardanaz E, Andersson A, Sund M, Ericson U, Wirfalt E, Key TJ, Travis RC, Gunter M, Riboli E, Vergnaud AC, Romieu I (2015) Healthy lifestyle and risk of breast cancer among postmenopausal women in the European Prospective Investigation into cancer and nutrition cohort study. Int J Cancer 136:2640-2648. https://doi.org/10.1002/ijc.29315

39. Dartois L, Fagherazzi G, Boutron-Ruault MC, Mesrine S, ClavelChapelon F (2014) Association between five lifestyle habits and cancer risk: results from the E3N cohort. Cancer Prev Res (Philadelphia, PA) 7:516-525. https://doi.org/10.1158/1940-6207.capr-13-0325

40. Gram IT, Lund-Larsen PG, Rosenlund AF, Stormer J (1989) Mammography screening in Tromso. Realization and results of the first mammography screening in Norway. Tidsskr Nor Laegeforen 109:1040-1042

41. Thomson CA, McCullough ML, Wertheim BC, Chlebowski RT, Martinez ME, Stefanick ML, Rohan TE, Manson JE, Tindle HA, Ockene J, Vitolins MZ, Wactawski-Wende J, Sarto GE, Lane DS, Neuhouser ML (2014) Nutrition and physical activity cancer prevention guidelines, cancer risk, and mortality in the women's health initiative. Cancer Prev Res (Philadelphia, PA) 7:42-53. https://doi.org/10.1158/1940-6207.capr-13-0258

42. Makarem N, Lin Y, Bandera EV, Jacques PF, Parekh N (2015) Concordance with World Cancer Research Fund/American Institute for Cancer Research (WCRF/AICR) guidelines for cancer prevention and obesity-related cancer risk in the Framingham Offspring cohort (1991-2008). Cancer Causes Control 26:277-286. https://doi.org/10.1007/s10552-014-0509-9

43. Catsburg C, Miller AB, Rohan TE (2014) Adherence to cancer prevention guidelines and risk of breast cancer. Int J Cancer 135:2444-2452. https://doi.org/10.1002/ijc.28887

44. Arthur R, Kirsh VA, Kreiger N, Rohan T (2018) A healthy lifestyle index and its association with risk of breast, endometrial, and ovarian cancer among Canadian women. Cancer Causes Control 29:485-493. https://doi.org/10.1007/s10552-018-1032-1

45. Harris HR, Bergkvist L, Wolk A (2016) Adherence to the World Cancer Research Fund/American Institute for Cancer Research recommendations and breast cancer risk. Int J Cancer 138:26572664. https://doi.org/10.1002/ijc.30015
46. Fund WCR, Research AIfC (2007) Food, nutrition, physical activity, and the prevention of cancer: a global perspective. American Institute for Cancer Research, New York

47. Tamimi RM, Spiegelman D, Smith-Warner SA, Wang M, Pazaris M, Willett WC, Eliassen AH, Hunter DJ (2016) Population attributable risk of modifiable and nonmodifiable breast cancer risk factors in postmenopausal breast cancer. Am J Epidemiol 184:884-893. https://doi.org/10.1093/aje/kww145

48. Ellingjord-Dale M, Vos L, Vik Hjerkind K, Hjartaker A, Russnes HG, Tretli S, Hofvind S, Dos-Santos-Silva I, Ursin G (2018) Number of risky lifestyle behaviors and breast cancer risk. JNCI Cancer Spectr 2:pky030. https://doi.org/10.1093/jncics/pky030

49. Collaborative Group on Hormonal Factors in Breast Cancer (2019) Type and timing of menopausal hormone therapy and breast cancer risk: individual participant meta-analysis of the worldwide epidemiological evidence. Lancet (London, England) 394:1159-1168. https://doi.org/10.1016/s0140-6736(19)31709-x

50. Pizot C, Boniol M, Mullie P, Koechlin A, Boniol M, Boyle P, Autier P (2016) Physical activity, hormone replacement therapy and breast cancer risk: a meta-analysis of prospective studies. Eur J Cancer (Oxford, England) 52:138-154. https://doi.org/10.1016/j.ejca.2015.10.063

51. McClain KM, McCullough LE, Bradshaw PT, Shantakumar S, Terry MB, Neugut AI, Gammon MD (2017) Age-specific indicators of a healthy lifestyle and postmenopausal breast cancer. J Women's Health 26:1176-1184. https://doi.org/10.1089/ jwh.2016.6028

52. Fund WCR (2018) Breast Cancer - How diet, nutrion and physical activity affect breast cancer risk. https://www.wcrf.org/sites/defau 1t/files/Breast-cancer-report.pdf

53. Gram IT, Park SY, Maskarinec G, Wilkens LR, Haiman CA, Le Marchand L (2019) Smoking and breast cancer risk by race/ethnicity and oestrogen and progesterone receptor status: the Multiethnic Cohort (MEC) study. Int J Epidemiol 48:501-511. https:// doi.org/10.1093/ije/dyy290

54. Arthur RS, Wang T, Xue X, Kamensky V, Rohan TE (2020) Genetic factors, adherence to healthy lifestyle behavior, and risk of invasive breast cancer among women in the UK Biobank. J Natl Cancer Inst. https://doi.org/10.1093/jnci/djz241

55. Macacu A, Autier P, Boniol M, Boyle P (2015) Active and passive smoking and risk of breast cancer: a meta-analysis. Breast Cancer Res Treat 154:213-224. https://doi.org/10.1007/s1054 9-015-3628-4

56. Azrad M, Blair CK, Rock CL, Sedjo RL, Wolin KY, DemarkWahnefried W (2019) Adult weight gain accelerates the onset of breast cancer. Breast Cancer Res Treat 176:649-656. https://doi. org/10.1007/s10549-019-05268-y

57. King MC, Marks JH, Mandell JB (2003) Breast and ovarian cancer risks due to inherited mutations in BRCA1 and BRCA2. Science (New York, NY) 302:643-646. https://doi.org/10.1126/scien ce. 1088759

58. Ayoub NM, Yaghan RJ, Abdo NM (2019) Impact of obesity on clinicopathologic characteristics and disease prognosis in pre- and postmenopausal breast cancer patients: a retrospective institutional study. J Obes 2019:3820759. https://doi.org/10.1155/2019/3820759

59. Picon-Ruiz M, Morata-Tarifa C, Valle-Goffin JJ, Friedman ER, Slingerland JM (2017) Obesity and adverse breast cancer risk and outcome: mechanistic insights and strategies for intervention. CA Cancer J Clin 67:378-397. https://doi.org/10.3322/caac.21405

60. Berrino F, Villarini A, Traina A, Bonanni B, Panico S, Mano MP, Mercandino A, Galasso R, Barbero M, Simeoni M, Bassi MC, Consolaro E, Johansson H, Zarcone M, Bruno E, Gargano G, Venturelli E, Pasanisi P (2014) Metabolic syndrome and breast cancer prognosis. Breast Cancer Res Treat 147:159-165. https:// doi.org/10.1007/s10549-014-3076-6

61. Simon MS, Beebe-Dimmer JL, Hastert TA (2018) Cardiometabolic risk factors and survival after breast cancer in the women's 
health initiative. Cancer 124:1798-1807. https://doi.org/10.1002/ cncr. 31230

62. de Haas EC, Oosting SF, Lefrandt JD, Wolffenbuttel BH, Sleijfer DT, Gietema JA (2010) The metabolic syndrome in cancer survivors. Lancet Oncol 11:193-203. https://doi.org/10.1016/s1470 -2045(09)70287-6

63. Johnson RH, Anders CK, Litton JK, Ruddy KJ, Bleyer A (2018) Breast cancer in adolescents and young adults. 65:e27397. https ://doi.org/10.1002/pbc.27397Pediatr Blood Cancer

64. Kirkham AA, Beaudry RI, Paterson DI, Mackey JR, Haykowsky MJ (2019) Curing breast cancer and killing the heart: a novel model to explain elevated cardiovascular disease and mortality risk among women with early stage breast cancer. Prog Cardiovasc Dis 62:116-126. https://doi.org/10.1016/j.pcad.2019.02.002

65. Chapman JA, Pritchard KI, Goss PE, Ingle JN, Muss HB, Dent SF, Vandenberg TA, Findlay B, Gelmon KA, Wilson CF, Shepherd LE, Pollak MN (2014) Competing risks of death in younger and older postmenopausal breast cancer patients. World J Clin Oncol 5:1088-1096. https://doi.org/10.5306/wjco.v5.i5.1088

66. Fougere B, Boulanger E, Nourhashemi F, Guyonnet S, Cesari M (2017) Chronic inflammation: accelerator of biological aging. J Gerontol Ser A 72:1218-1225. https://doi.org/10.1093/gerona/ glw240

67. Mantovani A, Allavena P, Sica A, Balkwill F (2008) Cancer-related inflammation. Nature 454:436-444. https://doi. org/10.1038/nature07205

68. Yu M, Hazelton WD (2019) Epigenetic aging: more than just a clock when it comes to cancer. Cancer Res. https://doi. org/10.1158/0008-5472.can-19-0924

69. Herrmann M, Pusceddu I, Marz W, Herrmann W (2018) Telomere biology and age-related diseases. Clin Chem Lab Med 56:1210 1222. https://doi.org/10.1515/cclm-2017-0870

70. Kim S, Parks CG, DeRoo LA, Chen H, Taylor JA, Cawthon RM, Sandler DP (2009) Obesity and weight gain in adulthood and telomere length. Cancer Epidemiol Biomark Prev 18:816-820. https ://doi.org/10.1158/1055-9965.epi-08-0935

71. Adraskela K, Veisaki E, Koutsilieris M, Philippou A (2017) Physical exercise positively influences breast cancer evolution. Clin Breast Cancer 17:408-417. https://doi.org/10.1016/j. clbc.2017.05.003

72. Wojcicki JM, Elwan D, Lin J, Blackburn E, Epel E (2018) Chronic obesity and incident hypertension in latina women are associated with accelerated telomere length loss over a 1-year period. Metab Syndr Relat Disord 16:262-266. https://doi.org/10.1089/ met.2017.0134

73. Latifovic L, Peacock SD, Massey TE, King WD (2016) The influence of alcohol consumption, cigarette smoking, and physical activity on leukocyte telomere length. Cancer Epidemiol Biomark Prev 25:374-380. https://doi.org/10.1158/1055-9965.epi-14-1364

74. Ennour-Idrissi K, Maunsell E, Diorio C (2017) Telomere length and breast cancer prognosis: a systematic review. Cancer Epidemiol Biomark Prev 26:3-10. https://doi.org/10.1158/1055-9965. epi-16-0343

75. Wang Z, Zhang Z, Guo Y, Shui H, Liu G, Jin T, Wang H (2018) Shorter telomere length is associated with increased breast cancer risk in a chinese han population: a case-control analysis. J Breast Cancer 21:391-398. https://doi.org/10.4048/jbc.2018.21.e52

76. Campa D, Barrdahl M, Santoro A, Severi G, Baglietto L, Omichessan H, Tumino R, Bueno-de-Mesquita HBA, Peeters PH, Weiderpass E, Chirlaque MD, Rodríguez-Barranco M, Agudo A, Gunter M, Dossus L, Krogh V, Matullo G, Trichopoulou A, Travis RC, Canzian F, Kaaks R (2018) Mitochondrial DNA copy number variation, leukocyte telomere length, and breast cancer risk in the European Prospective Investigation into cancer and nutrition (EPIC) study. Breast Cancer Res 20:29. https://doi.org/10.1186/ s13058-018-0955-5

77. Walter KR, Ford ME, Gregoski MJ, Kramer RM, Knight KD, Spruill L, Nogueira LM, Krisanits BA, Phan V, La Rue AC, Lilly MB, Ambs S, Chan K, Turner TF, Varner H, Singh S, Uribarri J, Garrett-Mayer E, Armeson KE, Hilton EJ, Clair MJ, Taylor MH, Abbott AM, Findlay VJ, Peterson LL, Magwood G, Turner DP (2019) Advanced glycation end products are elevated in estrogen receptor-positive breast cancer patients, alter response to therapy, and can be targeted by lifestyle intervention. Breast Cancer Res Treat 173:559-571. https://doi.org/10.1007/s10549-018-4992-7

78. Wilsgaard T, Jacobsen BK, Schirmer H, Thune I, Lochen ML, Njolstad I, Arnesen E (2001) Tracking of cardiovascular risk factors: the Tromso study, 1979-1995. Am J Epidemiol 154:418426. https://doi.org/10.1093/aje/154.5.418

79. van der Zee MD, van der Mee D, Bartels M, de Geus EJC (2019) Tracking of voluntary exercise behaviour over the lifespan. Int J Behav Nutr Phys Act 16:17. https://doi.org/10.1186/s1296 6-019-0779-4

80. Veerbeek MA, Ten Have M, van Dorsselaer SA, Oude Voshaar RC, Rhebergen D, Willemse BM (2019) Differences in alcohol use between younger and older people: results from a general population study. Drug Alcohol Depend 202:18-23. https://doi. org/10.1016/j.drugalcdep.2019.04.023

Publisher's Note Springer Nature remains neutral with regard to jurisdictional claims in published maps and institutional affiliations. 